\title{
Assessing village-level carbon balance due to greenhouse gas mitigation interventions using EX-ACT model
}

\author{
Ch. Srinivasarao ${ }^{1}$ Y. Sudha Rani ${ }^{1} \cdot$ V. Girija Veni ${ }^{1} \cdot$ K. L. Sharma ${ }^{1}$ • \\ G. R. Maruthi Sankar ${ }^{1}$ J. V. N. S. Prasad ${ }^{1}$-Y. G. Prasad ${ }^{1}$ K. L. Sahrawat ${ }^{2}$
}

Received: 10 January 2014/Revised: 19 February 2015 / Accepted: 9 March 2015/Published online: 9 April 2015

(C) Islamic Azad University (IAU) 2015

\begin{abstract}
Under National Initiative on Climate Resilient Agriculture (NICRA) project, a range of climate smart agricultural practices were evaluated with on-farm demonstrations during 2011-2013 in eight climatically vulnerable villages of Andhra Pradesh, India. Proven climate smart practices viz residue recycling, soil, water and nutrient management, afforestation and feeding + breeding practices in livestock were implemented in annual and perennial crops, irrigated rice, horticulture, fodder, forestry and livestock. An EX-ante carbon-balance tool (EX-ACT) developed by the FAO was used with a combination of various climate smart interventions to know the mitigation potentials in eight climatically vulnerable villages of Andhra Pradesh, India. Based on our observations, EXACT model had shown that these practices were effective to mitigate $\mathrm{CO}_{2}$ emissions apart from enhancing soil productivity. In Nacharam, Yagantipalli, Sirusuwada and Matsyapuri villages, climate smart practices implemented in annual crops along with crop residue recycling, crop and water management practices resulted in negative carbon (C) balance by $-16,410,-8851,-7271$ and $-6125 \mathrm{t} \mathrm{CO}_{2}$ $\mathrm{e}$, respectively. The EX-ACT model predicted positive carbon balance with irrigated module in the rice-growing villages of Sirusuwada and Matsyapuri villages. The negative values suggest a sink, and positive values a source for $\mathrm{CO}_{2}$ emissions. In Chamaluru village, although there
\end{abstract}

Ch. Srinivasarao

cherukumalli2011@gmail.com

1 Central Research Institute for Dryland Agriculture, Santhoshnagar, Saidabad (P.O.), Hyderabad 500 059, Andhra Pradesh, India

2 International Crops Research Institute for Semi Arid Tropics, Patancheru, Hyderabad 502 324, Andhra Pradesh, India were $\mathrm{CO}_{2}$ emissions (source) due to livestock and nonforest and land use changes, there was a carbon sink due to other activities as predicted by the model. The results suggested that various climatic smart practices at the village level were successful in creating net sink of $\mathrm{CO}_{2}$ emissions ( $\left.\mathrm{t} \mathrm{CO}_{2} \mathrm{e}\right)$.

Keywords $\mathrm{CO}_{2}$ emissions - EX-ACT model - Agriculture · Carbon balance $\cdot$ Climatic smart practices

\section{Introduction}

In the intergovernmental climate negotiations, India has consistently argued against greenhouse gases mitigation commitments for developing countries. India being 126th on the Human Development Index with $16 \%$ of world's population is now responsible for $5.1 \%$ of world's greenhouse gas (GHG) emissions. India's per capita emissions are 1.2 tons annually, which is far less than that of the most industrialized countries especially the USA and Canada with per capita rate of 19.8 and 17.5 metric tons, respectively (Rajamani 2007). Climate change is set to critically impair India's economic growth and its ability to meet with the development goals. The fourth assessment report of Intergovernmental Panel on Climate Change (IPCC) updates India's national programs to address the climate change. It identifies measures that promote developmental objectives and, at the same time, yielding cobenefits in addressing climate change effectively. It lists specific opportunities to simultaneously advance India's developmental and climate-related objectives of greenhouse gas mitigation.

Soil carbon sequestration is the process of transferring carbon dioxide from the atmosphere into soil in such a way 
that it results in a long-term increase in soil carbon stocks. This "sequestering" of carbon helps in enhancing soil quality and long-term agronomic productivity (Sundermeier et al. 2005), and this helps to sustain a good soil physical condition to absorb, retain and supply water and nutrients to crops. Soil organic carbon constitutes a significant component of the global terrestrial C pool (Lal 2010). Mitigation of carbon dioxide $\left(\mathrm{CO}_{2}\right)$ emission from agriculture can be achieved by increasing $\mathrm{C}$ sequestration in the soil, which implies storage of C as soil organic matter (Lal 2004). A high SOC can be achieved through adoption of appropriate crop rotations (Wright and Hons 2005), following integrated fertility management practices (Lal 2010; Srinivasarao et al. 2013) and using conservation tillage methods (Lal 2009). Adequate supply of nutrients in soil can improve soil organic C sequestration in tropical soils (Mandal et al. 2007; Srinivasarao et al. 2012). Addition of crop residues plays an important role in soil carbon sequestration in improving soil structure, soil water holding capacity and soil erosion prevention (Lal 2009). Methane emissions from lowland rice crop depend upon irrigation practices, organic matter content, temperature, fertilizer usage (Bhatia et al. 2005) and microbial processes of nitrification and denitrification. Livestock emissions of methane can be reduced by improved feed and feeding quality and management (Jordan et al. 2006) and by increasing the breeding efficiency of the animal. Application of manure could increase soil carbon by improving the soil fertility and soil structure and by improving soil $\mathrm{C}$ and nutrient pools. A balanced application of plant nutrients, organic amendments and inclusion of legumes in the cropping systems should enhance soil carbon stocks (Srinivasarao et al. 2011c).

The project "National Initiative on Climate resilient Agriculture" was initiated during 2010-11 by the Indian Government with an objective to implement climatic smart practices on farmers' fields for adapting to current climate risks. Under this, the impact of these practices on Ex-ante carbon balance was taken up in about 125 model villages in 100 climate-vulnerable districts of India in association with the Zonal Project Directorates (ZPD) and Krishi Vigyana Kendra (KVK). In this paper, we discuss the carbon balance for eight such villages in six climate-vulnerable districts in Andhra Pradesh, India. The magnitude of decline or enhancement of SOC due to continuous cultivation depends on the balance between loss of carbon and the quantity and quality of biomass that are returned and organic matter added to the soil (Srinivasarao et al. 2011a, b). Therefore, crop and soil management practices have to be adapted to sustain soil organic carbon. The aim of this study was to assess the carbon balance with implementation of climatic smart management practices related to agro-forestry, forestry, livestock and agriculture using EX-ante carbon-balance tool (EX-ACT) model in eight villages of Andhra Pradesh.

\section{Materials and methods}

\section{EX-ACT model}

Three FAO divisions (TCA, TCI and ESA) jointly developed the EX-ACT mainly to integrate the significant mitigation effects in agriculture and forestry development projects at the stage of project design. It was developed using the guidelines for national greenhouse gas inventories (IPCC, 2006) completed with existing methodologies (ADEME2, European environmental agency, AFD3, A/R $\mathrm{CDM}$, voluntary $\mathrm{C}$ markets) in order to be acceptable to the scientific community. For emissions of $\mathrm{CO}_{2}$ from energy consumption and all $\mathrm{N}_{2} \mathrm{O}$ and $\mathrm{CH}_{4}$ emissions, the generic approach considers multiplying an activity data (it can be area, animal numbers, mass unit or fuel quantity) by its specific emission factor for each source. For nonenergy-related $\mathrm{CO}_{2}$ emissions or sinks, most calculations, except if specified, use an approach with a stock-difference method: The emissions or sinks are calculated as the change over time of carbon stocks for the different pools. IPCC methods are based on five compartments: aboveground biomass, below-ground biomass, litter, dead-wood and soil carbon.

EX-ACT (http://www.fao.org/tc/exact/en/) is a landbased accounting system based on measuring $\mathrm{C}$ stocks, stock changes per unit of land, as well as methane $\left(\mathrm{CH}_{4}\right)$ and nitrous oxide $\left(\mathrm{N}_{2} \mathrm{O}\right)$ emissions expressed in tons of carbon dioxide equivalent per hectare $\left(\mathrm{t} \mathrm{CO}_{2} \mathrm{e} \mathrm{ha}^{-1}\right)$. The logic behind is based on the comparison between with and without project to know the extent of benefits in terms of $\mathrm{CO}_{2}$ mitigation. Ex-ante project evaluation compares the impacts of a planned intervention to the business-as-usual scenario. It is thus in the basic logic of EX-ACT that for the limited set of as-relevant identified variables, data are required for three points in time:

- The baseline situation

- The with-project scenario

- The without-project scenario (business-as-usual)

The main output of the tool consists of the $\mathrm{C}$ balance resulting from the difference between these two alternative scenarios (Fig. 1). Thus, $\mathrm{x}_{0}$ gives the initial situation of land use and management practices in the project area, e.g., the amount of cropland managed under improved nutrient management. The consecutively starting project intervention (with-project scenario) will lead to an increase in the improved managed area to $\mathrm{x}_{2}$. In the absence of the project intervention (without-project scenario), it is instead expected that this increase is smaller and only $\mathrm{x}_{1}$ hectares are managed in an improved way (cf. baseline scenario building). 


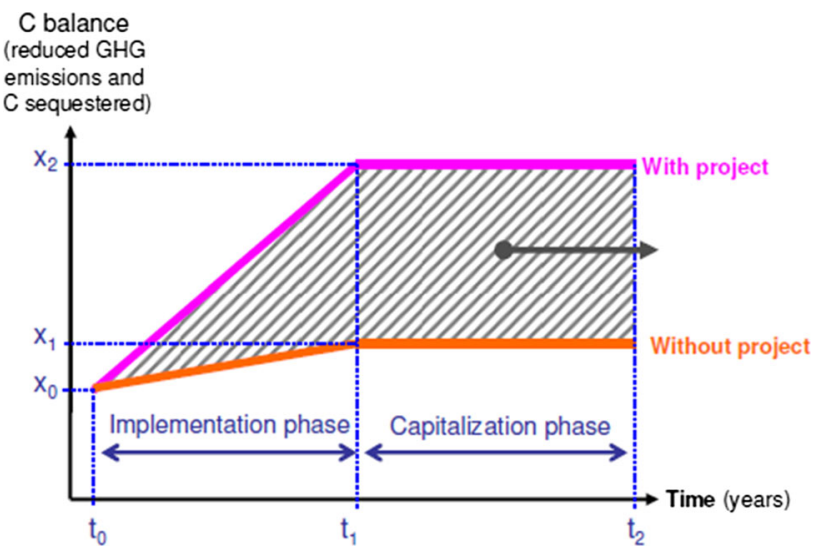

Fig. 1 Schematic representation of the methodology used to compute final C balance using EX-ACT (Source: Bernoux et al. 2010)

Thereby, EX-ACT differentiates between two time periods: The implementation phase defines the time period in which active project activities are carried out and lasts from $t_{0}$ until $t_{1}$. Thereby, the period covered by the analysis does not necessarily end with the termination of the active project intervention. Even after a new equilibrium in land use and practices is reached in $\mathrm{t} 1$, further changes may occur, e.g., in soil carbon content or in biomass, that are caused by the prior intervention. This period defines the capitalization phase which lasts from $\mathrm{t} 1$ until $\mathrm{t} 2$. The difference in activity data between with- and without-project scenarios serves then as the input data for calculating the carbon balance of the project. Usually, the sum of implementation and capitalization phases is set at 20 years. EXACT was designed to work at a project level, but it can easily be up-scaled at program/sector or national level (Bernoux et al. 2010).

The data needs in EX-ACT are very similar to the usual data required for ex-ante economic analyses of projects. Figure 2 illustrates this essential differentiation which is crucial to the correct understanding and application of EXACT andit (EX-ACT-Tool v 3.4) consists of a set of 18 linked Microsoft Excel sheets having (1) general description of the project (geographic area, climate and soil characteristics, duration of the project), (2) identification of changes in land use and technologies foreseen by project components (deforestation, afforestation/reforestation, annual/perennial crops, rice cultivation, grasslands, livestock, inputs, energy) and (3) computation of the $\mathrm{C}$ balance with and without the project using IPCC default values andwhen available—ad hoc coefficients.

\section{Selection of districts and village}

In Andhra Pradesh, eight villages in six districts: Nalgonda, Khammam, Anantapur, Kurnool, West Godavari and

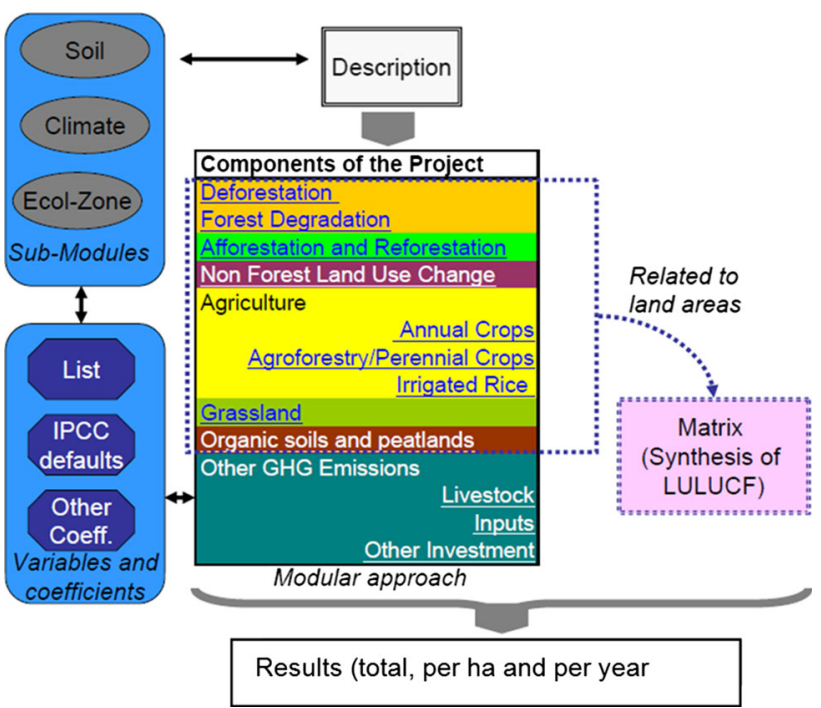

Fig. 2 Schematization of the modular EX-ACT structure (Source: Bernoux et al. 2010)

Srikakulam were selected under the NICRA project (Table 1) based on climatic vulnerabilities viz water scarcity, frequent drought, poor soil health, flood, moisture stress, water logging and mid-season drainage.

The major crops grown in the project-implemented villages are rice (Oryza sativa), cotton (Gossipium sp.), pigeon pea (Cajanus cajan), maize (Zea mays.), chili (Capsicum sp.), groundnut (Arachis hypogea), korra (Setaria italica), jowar (Sorghum sp.) and sunflower (Helianthus annuus). The farmers in these villages have grown the crops in traditional ${ }^{* 1}$ manner which have resulted in soil carbon loss. The practices in crop and soil management that result in decline of soil $\mathrm{C}$ storage include burning of crops residues, continuous cultivation, surface flood irrigation and indiscriminate use of fertilizers with little/scanty application of organic manures. Moreover, farmers adopted goat and sheep rearing to cope up with crop failures and frequent droughts, which results in emissions of GHGs.

Many practices which reduce vulnerability to climate variability have been introduced in the selected villages to improve the carbon balance through village-based climate smart management practices (improved ${ }^{* 2}$ ) and are summarized in Table 2.

The present analysis takes into account specific environmental features (soil and climate types) of each case study as well as key project activities with higher potential to affect $\mathrm{C}$ balance estimation. Average climate considered

\footnotetext{
${ }_{1}$ Traditional $^{*}=$ all the practices adopted by farmers before implementation of project

${ }^{2}$ Improved $*$ all the practices adopted by farmers after the implementation of project
} 
Table 1 Details of study villages under NICRA project in six districts of Andhra Pradesh, India

\begin{tabular}{|c|c|c|c|c|c|c|}
\hline District & Village & Latitude & Longitude & $\begin{array}{l}\text { Climate } \\
\text { vulnerability }\end{array}$ & $\begin{array}{l}\text { Area } \\
\text { (ha) }\end{array}$ & Crops grown \\
\hline \multirow[t]{2}{*}{ Nalgonda } & Nandylagudem & $\begin{array}{l}17^{\circ} 14^{\prime} \\
58.11^{\prime \prime}\end{array}$ & $\begin{array}{c}79^{\circ} 41^{\prime} \\
05.79^{\prime \prime}\end{array}$ & Drought & 194 & Cotton, pigeon pea, vegetables, rice \\
\hline & Boringthanda & $\begin{array}{r}17^{\circ} 16^{\prime} \\
09.62^{\prime \prime}\end{array}$ & $\begin{array}{l}79^{\circ} 40^{\prime} \\
35.60^{\prime \prime}\end{array}$ & Drought & 114 & Cotton, pigeon pea, vegetables, rice \\
\hline Khammam & Nacharam & $\begin{array}{r}17^{\circ} 20^{\prime} \\
00.39^{\prime \prime}\end{array}$ & $\begin{array}{l}80^{\circ} 28^{\prime} \\
54.31^{\prime \prime}\end{array}$ & $\begin{array}{l}\text { Drought and heat } \\
\text { stress }\end{array}$ & 1273 & Cotton, pigeon pea, chili, rice \\
\hline Kurnool & Yagantipalle & $\begin{array}{l}15^{\circ} 19^{\prime} \\
39.97^{\prime \prime}\end{array}$ & $\begin{array}{l}78^{\circ} 11^{\prime} \\
20.36^{\prime \prime}\end{array}$ & Drought & 542 & $\begin{array}{l}\text { Setaria, pigeon pea, sorghum, maize, sunflower, } \\
\text { vegetables }\end{array}$ \\
\hline \multirow[t]{2}{*}{ Anantapur } & Chamaluru & $\begin{array}{r}14^{\circ} 44^{\prime} \\
51.01^{\prime \prime}\end{array}$ & $\begin{array}{c}77^{\circ} 47^{\prime} \\
04.53^{\prime \prime}\end{array}$ & Drought & 2146 & Groundnut, chili, rice sweet orange, papaya \\
\hline & Chakrayapet & $\begin{array}{l}14^{\circ} 46^{\prime} \\
32.54^{\prime \prime}\end{array}$ & $\begin{array}{l}77^{\circ} 45^{\prime} \\
28.59^{\prime \prime}\end{array}$ & Drought & 173 & $\begin{array}{l}\text { Groundnut, castor, setaria, pigeon pea, rice, sweet } \\
\text { orange }\end{array}$ \\
\hline $\begin{array}{l}\text { West } \\
\text { Godavari }\end{array}$ & Matsyapuri & $\begin{array}{c}16^{\circ} 28^{\prime} \\
44.27^{\prime \prime}\end{array}$ & $\begin{array}{l}81^{\circ} 37^{\prime} \\
40.95^{\prime \prime}\end{array}$ & Cyclone & 630 & Rice, blackgram \\
\hline Srikakulam & Sirusuwada & $\begin{array}{c}18^{\circ} 48^{\prime} \\
05.64^{\prime \prime}\end{array}$ & $\begin{array}{l}83^{\circ} 53^{\prime} \\
55.66^{\prime \prime}\end{array}$ & Floods & 326 & Rice, blackgram, groundnut, sesame \\
\hline
\end{tabular}

in the analysis is warm tropical with a mean annual temperature equal to $22{ }^{\circ} \mathrm{C}$, while the dominant soil type considered was highly active clay (HAC) dominated by $2: 1$ clay minerals. The moisture regime is dry for Nalgonda, Khammam, Kurnool and Ananthapur districts and moist for Srikakulam and West Godavari districts. Such information is essential, as most coefficients used in the analysis can change drastically according to soil characteristics and climate conditions. The input required for model simulation was collected based on the survey conducted by taking the village as a unit and through interactions with the farmers in these villages. The analysis is based on a comparison between the $\mathrm{C}$ balance in the "with-project" scenario and the C balance "without-project" scenario. The "with-project" case is built by taking into account the activities undertaken under the NICRA project.

\section{Statistical analysis}

Descriptive statistical techniques were employed in data analysis, while linear regression was used to determine the relationship among livestock and small ruminants, area under annual crops and methane emissions in irrigated rice with $\mathrm{C}$ balance. The level of significance was tested at $p=0.05$.

\section{Results and discussion}

\section{Carbon balance}

The EX-ACT model was simulated in eight villages of Andhra Pradesh, India, with different technological interventions to estimate the carbon balance in the target villages. In Nandyalagudem, Nacharam and Yagantipalli, the model projected the carbon (C) balance of -5289 , 12,860 and $-19,238 \mathrm{t} \mathrm{CO}_{2} \mathrm{e}$, respectively. The negative values suggest that there was a reduction in $\mathrm{CO}_{2}$ emissions (sink). This reduction in $\mathrm{CO}_{2}$ emissions is mainly due to adoption of climate smart agricultural practices in different production systems. However, model projected $\mathrm{C}$ balance of $-9362 \mathrm{t} \mathrm{CO}_{2}$ e (Fig. 3) in Chamaluru, mainly due to adoption of interventions in inputs (chemical fertilizers) management. In Matsyapuri and Sirusuwada villages where rice is the predominant crop, the model projected $\mathrm{C}$ balance of $-19,201$ and $-3990 \mathrm{t} \mathrm{CO}_{2}$ e. In this study, model projected negative carbon balance due to the implementation of different climate smart technological interventions in annual, perennial crops and use of chemical fertilizers according to the crop demand and implementing breeding and feeding management practices in livestock and small ruminants in the study villages. It was found that 
Table 2 Climate smart management practices implemented in eight villages as a part of NICRA project

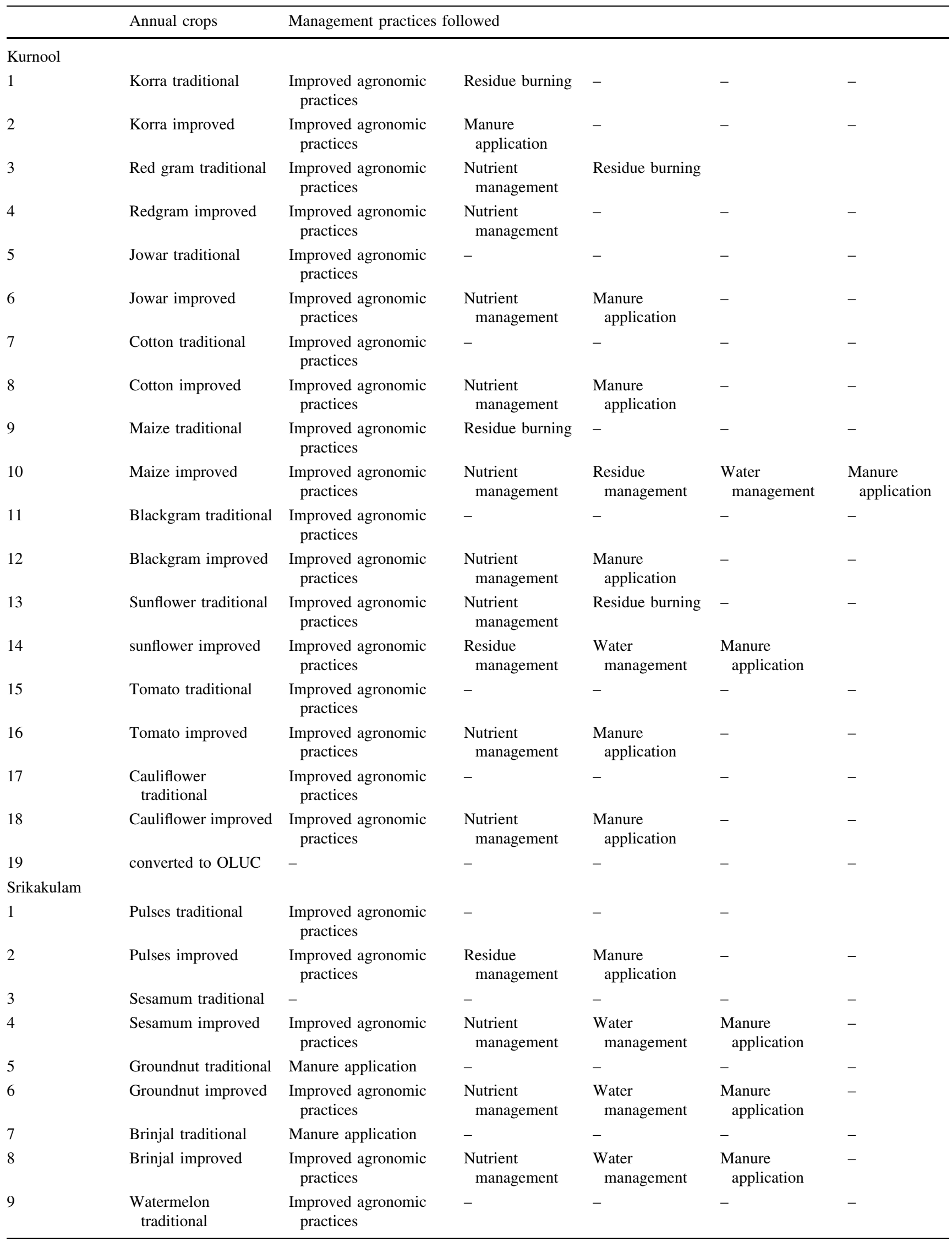


Table 2 continued

\begin{tabular}{|c|c|c|c|c|c|c|}
\hline & Annual crops & Management practice & llowed & & & \\
\hline 10 & $\begin{array}{c}\text { Watermelon } \\
\text { improved }\end{array}$ & $\begin{array}{l}\text { Improved agronomic } \\
\text { practices }\end{array}$ & $\begin{array}{l}\text { Nutrient } \\
\text { management }\end{array}$ & $\begin{array}{l}\text { Manure } \\
\text { application }\end{array}$ & - & - \\
\hline 11 & Pulses from paddy & $\begin{array}{l}\text { Improved agronomic } \\
\text { practices }\end{array}$ & $\begin{array}{l}\text { Residue } \\
\text { management }\end{array}$ & - & - & - \\
\hline 12 & Annual from OLUC & $\begin{array}{l}\text { Improved agronomic } \\
\text { practices }\end{array}$ & $\begin{array}{l}\text { Nutrient } \\
\text { management }\end{array}$ & $\begin{array}{l}\text { Water } \\
\text { management }\end{array}$ & $\begin{array}{l}\text { Manure } \\
\text { application }\end{array}$ & - \\
\hline Boringthanda & & & & & & - \\
\hline 1 & Cotton traditional & $\begin{array}{l}\text { Improved agronomic } \\
\text { practices }\end{array}$ & Residue burning & - & - & - \\
\hline 2 & Cotton improved & $\begin{array}{l}\text { Improved agronomic } \\
\text { practices }\end{array}$ & $\begin{array}{l}\text { Nutrient } \\
\text { management }\end{array}$ & $\begin{array}{l}\text { Water } \\
\text { management }\end{array}$ & $\begin{array}{l}\text { Manure } \\
\text { application }\end{array}$ & - \\
\hline 3 & Redgram traditional & Residue burning & - & - & - & - \\
\hline 4 & Redgram improved & $\begin{array}{l}\text { Improved agronomic } \\
\text { practices }\end{array}$ & $\begin{array}{l}\text { Nutrient } \\
\text { management }\end{array}$ & $\begin{array}{l}\text { Water } \\
\text { management }\end{array}$ & - & - \\
\hline 5 & $\begin{array}{l}\text { Greengram } \\
\text { traditional }\end{array}$ & $\begin{array}{l}\text { Improved agronomic } \\
\text { practices }\end{array}$ & - & - & - & - \\
\hline 6 & Greengram improved & $\begin{array}{l}\text { Improved agronomic } \\
\text { practices }\end{array}$ & $\begin{array}{l}\text { Nutrient } \\
\text { management }\end{array}$ & $\begin{array}{l}\text { Water } \\
\text { management }\end{array}$ & $\begin{array}{l}\text { Residue } \\
\text { management }\end{array}$ & - \\
\hline 7 & Vegetables traditional & $\begin{array}{l}\text { Improved agronomic } \\
\text { practices }\end{array}$ & - & - & - & - \\
\hline 8 & Vegetables improved & $\begin{array}{l}\text { Improved agronomic } \\
\text { practices }\end{array}$ & $\begin{array}{l}\text { Nutrient } \\
\text { management }\end{array}$ & $\begin{array}{l}\text { Water } \\
\text { management }\end{array}$ & $\begin{array}{l}\text { Manure } \\
\text { application }\end{array}$ & - \\
\hline 9 & Chili traditional & $\begin{array}{l}\text { Improved agronomic } \\
\text { practices }\end{array}$ & - & - & - & - \\
\hline 10 & Chili improved & $\begin{array}{l}\text { Improved agronomic } \\
\text { practices }\end{array}$ & $\begin{array}{l}\text { Nutrient } \\
\text { management }\end{array}$ & $\begin{array}{l}\text { Water } \\
\text { management }\end{array}$ & $\begin{array}{l}\text { Manure } \\
\text { application }\end{array}$ & - \\
\hline Nandyalagudem & & & & & & - \\
\hline 1 & Cotton traditional & $\begin{array}{l}\text { Improved agronomic } \\
\text { practices }\end{array}$ & Residue burning & - & - & - \\
\hline 2 & Cotton improved & $\begin{array}{l}\text { Improved agronomic } \\
\text { practices }\end{array}$ & $\begin{array}{l}\text { Nutrient } \\
\text { management }\end{array}$ & $\begin{array}{l}\text { Water } \\
\text { management }\end{array}$ & $\begin{array}{l}\text { Manure } \\
\text { application }\end{array}$ & - \\
\hline 3 & $\begin{array}{l}\text { Greengram } \\
\text { traditional }\end{array}$ & $\begin{array}{l}\text { Improved agronomic } \\
\text { practices }\end{array}$ & - & - & - & - \\
\hline 4 & Greengram improved & $\begin{array}{l}\text { Improved agronomic } \\
\text { practices }\end{array}$ & $\begin{array}{l}\text { Residue } \\
\text { management }\end{array}$ & $\begin{array}{l}\text { Manure } \\
\text { application }\end{array}$ & - & - \\
\hline 5 & Chili traditional & $\begin{array}{l}\text { Improved agronomic } \\
\text { practices }\end{array}$ & - & - & - & - \\
\hline 6 & Chili improved & $\begin{array}{l}\text { Improved agronomic } \\
\text { practices }\end{array}$ & $\begin{array}{l}\text { Nutrient } \\
\text { management }\end{array}$ & $\begin{array}{l}\text { Water } \\
\text { management }\end{array}$ & $\begin{array}{l}\text { Manure } \\
\text { application }\end{array}$ & - \\
\hline 7 & Redgram traditional & $\begin{array}{l}\text { Improved agronomic } \\
\text { practices }\end{array}$ & $\begin{array}{l}\text { Nutrient } \\
\text { management }\end{array}$ & Residue burning & - & - \\
\hline 8 & Redgram improved & $\begin{array}{l}\text { Improved agronomic } \\
\text { practices }\end{array}$ & - & - & - & - \\
\hline 9 & Redgram traditional & $\begin{array}{l}\text { Improved agronomic } \\
\text { practices }\end{array}$ & $\begin{array}{l}\text { Nutrient } \\
\text { management }\end{array}$ & - & - & - \\
\hline 10 & Redgram to cotton & $\begin{array}{l}\text { Improved agronomic } \\
\text { practices }\end{array}$ & $\begin{array}{l}\text { Nutrient } \\
\text { management }\end{array}$ & $\begin{array}{l}\text { Manure } \\
\text { application }\end{array}$ & - & - \\
\hline 11 & Maize traditional & $\begin{array}{l}\text { Improved agronomic } \\
\text { practices }\end{array}$ & - & - & - & - \\
\hline 12 & Maize improved & $\begin{array}{l}\text { Improved agronomic } \\
\text { practices }\end{array}$ & $\begin{array}{l}\text { Nutrient } \\
\text { management }\end{array}$ & $\begin{array}{l}\text { Water } \\
\text { management }\end{array}$ & $\begin{array}{l}\text { Residue } \\
\text { management }\end{array}$ & - \\
\hline Chamaluru & & & & & & - \\
\hline 1 & Groundnut traditional & $\begin{array}{l}\text { Improved agronomic } \\
\text { practices }\end{array}$ & $\begin{array}{l}\text { Manure } \\
\text { application }\end{array}$ & - & - & - \\
\hline
\end{tabular}


Table 2 continued

\begin{tabular}{|c|c|c|c|c|c|c|}
\hline & Annual crops & Management practices & llowed & & & \\
\hline 2 & Groundnut improved & $\begin{array}{l}\text { Improved agronomic } \\
\text { practices }\end{array}$ & $\begin{array}{l}\text { Nutrient } \\
\text { management }\end{array}$ & $\begin{array}{l}\text { Water } \\
\text { management }\end{array}$ & $\begin{array}{l}\text { Manure } \\
\text { application }\end{array}$ & - \\
\hline 3 & Redgram traditional & $\begin{array}{l}\text { Improved agronomic } \\
\text { practices }\end{array}$ & $\begin{array}{l}\text { Manure } \\
\text { application }\end{array}$ & Residue burning & - & - \\
\hline 4 & Redgram improved & $\begin{array}{l}\text { Improved agronomic } \\
\text { practices }\end{array}$ & $\begin{array}{l}\text { Nutrient } \\
\text { management }\end{array}$ & $\begin{array}{l}\text { Manure } \\
\text { application }\end{array}$ & Residue burning & - \\
\hline 5 & Tomato traditional & $\begin{array}{l}\text { Improved agronomic } \\
\text { practices }\end{array}$ & $\begin{array}{l}\text { Manure } \\
\text { application }\end{array}$ & - & - & - \\
\hline 6 & Tomato improved & $\begin{array}{l}\text { Improved agronomic } \\
\text { practices }\end{array}$ & $\begin{array}{l}\text { Nutrient } \\
\text { management }\end{array}$ & $\begin{array}{l}\text { Water } \\
\text { management }\end{array}$ & $\begin{array}{l}\text { Manure } \\
\text { application }\end{array}$ & - \\
\hline 7 & $\begin{array}{c}\text { Watermelon } \\
\text { traditional }\end{array}$ & $\begin{array}{l}\text { Improved agronomic } \\
\text { practices }\end{array}$ & $\begin{array}{l}\text { Manure } \\
\text { application }\end{array}$ & - & - & - \\
\hline 8 & $\begin{array}{c}\text { Watermelon } \\
\text { improved }\end{array}$ & $\begin{array}{l}\text { Improved agronomic } \\
\text { practices }\end{array}$ & $\begin{array}{l}\text { Water } \\
\text { management }\end{array}$ & - & - & - \\
\hline 9 & Chili traditional & $\begin{array}{l}\text { Improved agronomic } \\
\text { practices }\end{array}$ & $\begin{array}{l}\text { Manure } \\
\text { application }\end{array}$ & - & - & - \\
\hline 10 & Chili improved & $\begin{array}{l}\text { Improved agronomic } \\
\text { practices }\end{array}$ & $\begin{array}{l}\text { Nutrient } \\
\text { management }\end{array}$ & $\begin{array}{l}\text { Water } \\
\text { management }\end{array}$ & $\begin{array}{l}\text { Manure } \\
\text { application }\end{array}$ & - \\
\hline 11 & Brinjal traditional & $\begin{array}{l}\text { Improved agronomic } \\
\text { practices }\end{array}$ & - & - & - & - \\
\hline 12 & Brinjal improved & $\begin{array}{l}\text { Improved agronomic } \\
\text { practices }\end{array}$ & - & - & - & - \\
\hline 13 & Annual from OLUC & $\begin{array}{l}\text { Improved agronomic } \\
\text { practices }\end{array}$ & $\begin{array}{l}\text { Nutrient } \\
\text { management }\end{array}$ & $\begin{array}{l}\text { Manure } \\
\text { application }\end{array}$ & Residue burning & - \\
\hline \multicolumn{7}{|c|}{ Chakrayapeta } \\
\hline 1 & Groundnut traditional & $\begin{array}{l}\text { Improved agronomic } \\
\text { practices }\end{array}$ & $\begin{array}{l}\text { Water } \\
\text { management }\end{array}$ & $\begin{array}{l}\text { Manure } \\
\text { application }\end{array}$ & - & - \\
\hline 2 & Groundnut improved & $\begin{array}{l}\text { Improved agronomic } \\
\text { practices }\end{array}$ & $\begin{array}{l}\text { Nutrient } \\
\text { management }\end{array}$ & $\begin{array}{l}\text { Water } \\
\text { management }\end{array}$ & $\begin{array}{l}\text { Manure } \\
\text { application }\end{array}$ & - \\
\hline 3 & $\begin{array}{l}\text { castor from } \\
\text { groundnut }\end{array}$ & $\begin{array}{l}\text { Improved agronomic } \\
\text { practices }\end{array}$ & $\begin{array}{l}\text { Manure } \\
\text { application }\end{array}$ & - & - & - \\
\hline 4 & Redgram traditional & $\begin{array}{l}\text { Improved agronomic } \\
\text { practices }\end{array}$ & Residue burning & - & - & - \\
\hline 5 & Redgram improved & $\begin{array}{l}\text { Improved agronomic } \\
\text { practices }\end{array}$ & $\begin{array}{l}\text { Nutrient } \\
\text { management }\end{array}$ & $\begin{array}{l}\text { Manure } \\
\text { application }\end{array}$ & - & - \\
\hline 6 & $\begin{array}{l}\text { Korra from } \\
\text { groundnut }\end{array}$ & $\begin{array}{l}\text { Improved agronomic } \\
\text { practices }\end{array}$ & $\begin{array}{l}\text { Manure } \\
\text { application }\end{array}$ & - & - & - \\
\hline 7 & $\begin{array}{l}\text { Vegetables from } \\
\text { groundnut }\end{array}$ & $\begin{array}{l}\text { Improved agronomic } \\
\text { practices }\end{array}$ & $\begin{array}{l}\text { Nutrient } \\
\text { management }\end{array}$ & $\begin{array}{l}\text { Water } \\
\text { management }\end{array}$ & - & - \\
\hline 8 & Annual from OLUC & $\begin{array}{l}\text { Improved agronomic } \\
\text { practices }\end{array}$ & $\begin{array}{l}\text { Water } \\
\text { management }\end{array}$ & - & - & - \\
\hline \multicolumn{7}{|c|}{ West Godavari } \\
\hline 1 & Rice fallow pulses & Residue management & - & - & - & - \\
\hline \multicolumn{7}{|c|}{ Nacharam } \\
\hline 1 & Cotton traditional & $\begin{array}{l}\text { Improved agronomic } \\
\text { practices }\end{array}$ & $\begin{array}{l}\text { Nutrient } \\
\text { management }\end{array}$ & $\begin{array}{l}\text { Water } \\
\text { management }\end{array}$ & Residue burning & - \\
\hline 2 & Cotton improved & $\begin{array}{l}\text { Improved agronomic } \\
\text { practices }\end{array}$ & $\begin{array}{l}\text { Nutrient } \\
\text { management }\end{array}$ & $\begin{array}{l}\text { Water } \\
\text { management }\end{array}$ & $\begin{array}{l}\text { Manure } \\
\text { application }\end{array}$ & - \\
\hline 3 & Chili traditional & $\begin{array}{l}\text { Improved agronomic } \\
\text { practices }\end{array}$ & - & - & - & - \\
\hline 4 & Chili improved & $\begin{array}{l}\text { Improved agronomic } \\
\text { practices }\end{array}$ & $\begin{array}{l}\text { Nutrient } \\
\text { management }\end{array}$ & $\begin{array}{l}\text { Water } \\
\text { management }\end{array}$ & $\begin{array}{l}\text { Manure } \\
\text { application }\end{array}$ & - \\
\hline 5 & Redgram traditional & $\begin{array}{l}\text { Improved agronomic } \\
\text { practices }\end{array}$ & $\begin{array}{l}\text { Nutrient } \\
\text { management }\end{array}$ & Residue burning & - & - \\
\hline
\end{tabular}


Table 2 continued

\begin{tabular}{|c|c|c|c|c|c|c|}
\hline & Annual crops & Management practice & llowed & & & \\
\hline 6 & Redgram improved & $\begin{array}{l}\text { Improved agronomic } \\
\text { practices }\end{array}$ & $\begin{array}{l}\text { Nutrient } \\
\text { management }\end{array}$ & $\begin{array}{l}\text { Water } \\
\text { management }\end{array}$ & - & - \\
\hline 7 & $\begin{array}{l}\text { Greengram } \\
\text { traditional }\end{array}$ & $\begin{array}{l}\text { Improved agronomic } \\
\text { practices }\end{array}$ & - & - & - & - \\
\hline 8 & Greengram improved & $\begin{array}{l}\text { Improved agronomic } \\
\text { practices }\end{array}$ & $\begin{array}{l}\text { Nutrient } \\
\text { management }\end{array}$ & $\begin{array}{l}\text { Residue } \\
\text { management }\end{array}$ & - & - \\
\hline 9 & Vegetables traditional & - & - & - & - & - \\
\hline 10 & Vegetables improved & $\begin{array}{l}\text { Improved agronomic } \\
\text { practices }\end{array}$ & $\begin{array}{l}\text { Nutrient } \\
\text { management }\end{array}$ & $\begin{array}{l}\text { Manure } \\
\text { application }\end{array}$ & - & - \\
\hline
\end{tabular}

Traditional management practices followed by farmers before the implementation of project

Improved management practices followed by farmers after the implementation of project

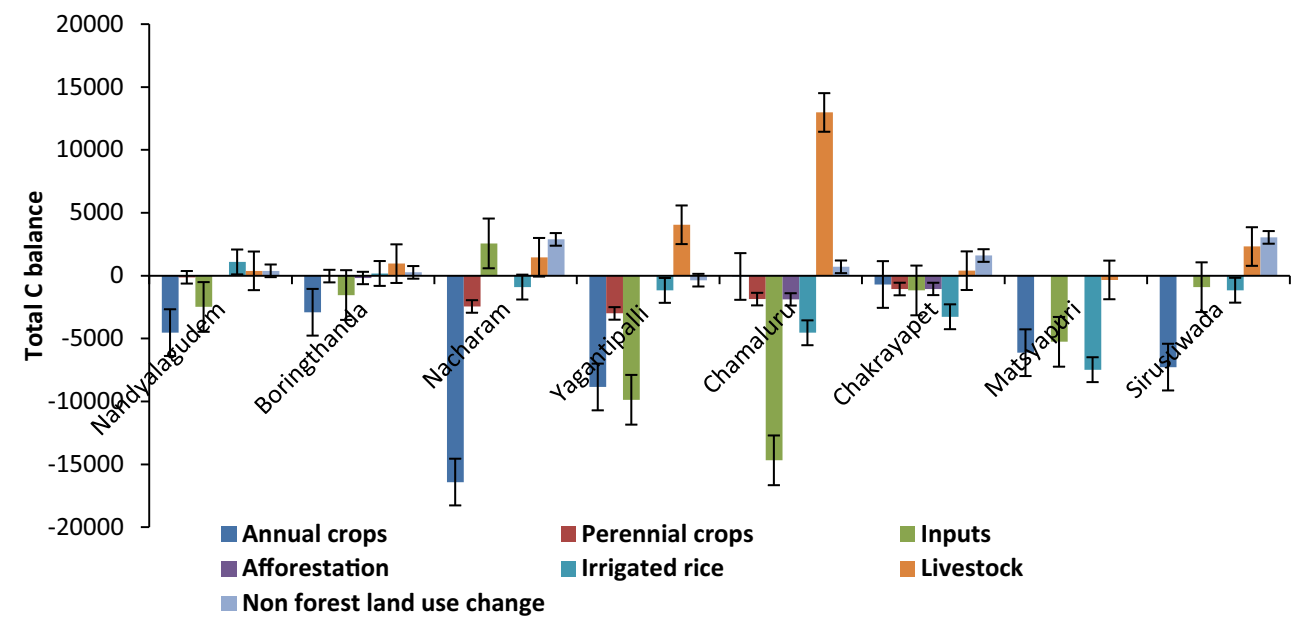

Fig. 3 Effect of different climate smart management practices on $\mathrm{C}$ balance $\left(\mathrm{t} \mathrm{CO}_{2} \mathrm{e}\right)$ in eight locations (- values indicates net sink and + value indicates net emissions)

positive values of $\mathrm{C}$ balance suggest a source for $\mathrm{CO}_{2}$ emissions where it is necessary to take measures that check the $\mathrm{CO}_{2}$ emissions. In our study, positive values of $\mathrm{C}$ balance were seen in the livestock and small ruminant's management in most of study villages although farmers adopted feeding practices for only $60 \%$ and breeding management practices in only $40 \%$ of the total livestock and small ruminants mainly due to an increase in the livestock population with the implementation of the project. Also, $\mathrm{C}$ balance became positive in land use change in all the villages except Yagantipalle where there was a reduction in $\mathrm{CO}_{2}$ emissions, suggesting that there can be emission or mitigation of $\mathrm{CO}_{2}$ emissions according to the land use change.

\section{Improved annual crop management practices}

Two scenarios "with project" and "without project" were studied mainly to know the difference in $\mathrm{C}$ balance with existing farmers practice (without project) and after adoption of different climate smart management practices in annual crops (with project), e.g., use of improved varieties, water management practices, manure management and application of fertilizers based on soil testing. All the practices followed by the farmers without and with project in all the crops grown in study villages are shown in Table 2 as traditional (without project and improved (with project). In Nacharam, model projected mitigation of $\mathrm{CO}_{2}$ emissions with a $\mathrm{C}$ balance of $-23,953 \mathrm{t} \mathrm{CO}_{2} \mathrm{e}$ "with" 
Table 3 Change in $\mathrm{C}$ balance by adoption of climate smart management practices in annual crops

\begin{tabular}{lrrr}
\hline Village & \multicolumn{2}{c}{ C balance } & Change in C balance \\
\cline { 2 - 3 } & Without project & With project & \\
\hline Nacharam & -7543 & $-23,953$ & $-16,410$ \\
Nandyalagudem & 834 & -4528 & -5362 \\
Boringthanda & 434 & -2477 & -2911 \\
Srikakulam & -3140 & $-10,270$ & -7130 \\
Yagantipalli & 368 & -8482 & -8850 \\
Matsyapuri & 0 & -6125 & -6125 \\
Chamaluru & $-54,020$ & $-54,085$ & -65 \\
Chakrayapet & -2368 & 3070 & -703 \\
Min & $-54,020$ & $-54,085$ & $-16,410$ \\
Max & 834 & 3070 & -65 \\
Mean & -8179 & $-13,356$ & -5945 \\
SD & 18,732 & 18,216 & 5223 \\
CV & -229 & -136 & -88 \\
\hline
\end{tabular}

- Carbon sink and + carbon source project and $-7543 \mathrm{t} \mathrm{CO}_{2} \mathrm{e}$ "without" project in annual crops (Table 3). The model predicted that "with project" C balance was $-10,270 \mathrm{t} \mathrm{CO}_{2}$ e and "without" project was 3140 t CO$_{2}$ e in Sirusuwada village, where 80 ha of pulse crops were grown. By adopting the climate smart management practices, it was observed that the $\mathrm{CO}_{2}$ emissions were mitigated at the rate of $0.61,0.93,1.06$ and $1.42 \mathrm{t}$ $\mathrm{CO}_{2} \mathrm{ha}^{-1}$ year $^{-1}$ in Mattsyapuri, Nacharam, Yagantipalli and Nandyalagudem villages, respectively. There existed a gap between with and without the project with respect to $\mathrm{CO}_{2}$ emissions in different study villages. Based on our study, although $\mathrm{CO}_{2}$ emissions were mitigated under traditional practice (without project), the extent of its mitigation was increased to a greater degree by following climate smart management practices, i.e., with improved practice (with project). Based on the model projections for the data, the rate of sink for $\mathrm{CO}_{2}$ emissions was higher in the improved practice compared to the traditional practice at different study villages. In majority of crops grown in different study villages, the change in total area was between 100 and 800 ha, while the change in $\mathrm{C}$ balance was in the range of $-16,410$ to $-65 \mathrm{t} \mathrm{CO}_{2} \mathrm{e}$.

\section{Improved or water saving paddy systems}

Rice cultivation is an important source of $\mathrm{CH}_{4}$ and $\mathrm{N}_{2} \mathrm{O}$. Rice crop was mainly grown in Matsyapuri (616 ha), Chamaluru (292 ha), Sirusuwada (140 ha) villages,
Table 4 Methane emitted in terms $\mathrm{CO}_{2}$ emissions $\left(\mathrm{t} \mathrm{CO}_{2}\right.$ e) during the rice cultivation

\begin{tabular}{lrrr}
\hline Village & Without project & With project & Balance \\
\hline Matsyapuri & 89,837 & 82,362 & -7475 \\
Sirusuwada & 10,129 & 8971 & -1158 \\
Yagantipalli & 7341 & 6169 & -1172 \\
Nacharam & 7236 & 6328 & -908 \\
Chamaluru & 31,843 & 27,300 & -4542 \\
Chakrayapet & 5735 & 2468 & -3268 \\
Min & 5735 & 2468 & -7475 \\
Max & 89,837 & 82,362 & -908 \\
Mean & 25,354 & 22,266 & -3087 \\
SD & 33,074 & 30,720 & 2590 \\
CV & 130 & 138 & -84 \\
\hline
\end{tabular}

- Carbon sink and + carbon source

followed by Yagatipalli (72 ha) village. 'Without' project rice crop was grown under continuous flooding irrigation, which resulted in methane emissions of $89,837,31,843$, 10,129 and $7341 \mathrm{t} \mathrm{CO}_{2}$ e, respectively. "With" project, adopting the interventions in water management practices, e.g., intermittent flooding irrigation, incorporation of green manure and straw into the soil, the methane emissions was mitigated (Table 4). In Matsyapuri, intermittent flooding along with incorporation of green manure and straw has mitigated methane emissions by $82,362 \mathrm{t} \mathrm{CO}_{2} \mathrm{e}$ 
Table 5 Carbon balance $\left(\mathrm{t} \mathrm{CO}_{2}\right.$ e) through afforestation and cultivation of perennial crops

\begin{tabular}{lccl}
\hline Village & Without project & With project & Carbon balance \\
\hline Afforestation & & & \\
Chamaluru & 0 & -1886 & -1886 \\
Chakrayapet & 0 & -1056 & -1056 \\
Perennial crops & & & \\
Nacharam & -1435 & -3885 & -2450 \\
Yagantipalli & -363 & -3365 & -3002 \\
Chamaluru & -363 & -2228 & -1865 \\
\hline
\end{tabular}

- Carbon sink and + carbon source

and created a $\mathrm{C}$ balance of $-7475 \mathrm{t} \mathrm{CO}_{2}$ e. In Chamaluru, the irrigated rice module of EX-ACT model projected that intermittent flooding and addition of organic amendments (green manure incorporation) resulted in $\mathrm{CO}_{2}$ emissions of $27,300 \mathrm{t} \mathrm{CO}_{2} \mathrm{e}$ "with" project and created a $\mathrm{C}$ balance of $-4542 \mathrm{t} \mathrm{CO}_{2}$ e. In Sirusuwada, the farmers grew rice crop with intermittent flooding and incorporation of straw into the field resulted in reduction in methane emissions in terms of $\mathrm{CO}_{2}$ emissions with a $\mathrm{C}$ balance of $1158 \mathrm{t}$ $\mathrm{CO}_{2}$ e. In Yagantipalli, the irrigated module in EX-ACT model projected that there was methane emissions in terms of $\mathrm{CO}_{2}$ emissions by $6169 \mathrm{t} \mathrm{CO}_{2} \mathrm{e}$ with intermittent flooded irrigation and green manure incorporation in field and created a $\mathrm{C}$ balance of $-1172 \mathrm{t} \mathrm{CO}_{2} \mathrm{e}$. The total $\mathrm{C}$ balance for paddy system was found to be in the range of -908 to $-7475 \mathrm{t} \mathrm{CO}_{2}$ e based on the study.

\section{Afforestation and perennial crops}

In Chamaluru and Chakrayapet, about 5 and 2.8 ha wasteland was brought under afforestation, and this resulted in change in $\mathrm{C}$ balance. Eucalyptus globus and Acacia nilotica plantation were able to mitigate $\mathrm{CO}_{2}$ emissions by -1886 and $-1056 \mathrm{t} \mathrm{CO}_{2}$ e (Table 5). It was observed that by adopting afforestation, $\mathrm{CO}_{2}$ emissions were mitigated at the rate of $18.8 \mathrm{t} \mathrm{CO}_{2} \mathrm{ha}^{-1}$ year $^{-1} \mathrm{C}$. Due to lack of profitable commercial prices for crops such as cotton and chili, farmers are interested to grow perennial crops such as mango, citrus, sweet orange and guava. As a result 14, 20 and 16 ha of area under annual crops were converted into perennial crops in Nacharam, Yagantipalli and Chamaluru villages, respectively. In Nacharam, perennial crops resulted in reducing the $\mathrm{CO}_{2}$ emissions by $-2450 \mathrm{tCO}_{2}$ e (Table 5). In Yagantipalli, the cultivation of papaya and mango instead of annual crops resulted in the mitigation of $\mathrm{CO}_{2}$ emissions by $-3002 \mathrm{t} \mathrm{CO}_{2}$
Table 6 Carbon balance ( $\mathrm{CO}_{2}$ e) in non-forest land use change using EX-ACT model

\begin{tabular}{llrc}
\hline Village & Without & With & Carbon balance \\
\hline Nandyalagudem & 0 & 384 & 384 \\
Boringthanda & 0 & 261 & 261 \\
Nacharam & 0 & 3566 & 3566 \\
Yagantipalli & 0 & -359 & -359 \\
Sirusuwada & 0 & 3042 & 3042 \\
Chamaluru & 0 & 703 & 703 \\
Chakrayapet & 0 & 1600 & 1600
\end{tabular}

- Carbon sink and + carbon source

e. In Chamaluru, area under annual crops was converted into sweet orange and guava and showed mitigation of $\mathrm{CO}_{2}$ emissions by $-1865 \mathrm{t} \mathrm{CO}_{2}$ e. It was observed that $\mathrm{CO}_{2}$ emissions were mitigated at the rate of $8.8,7.5$ and $5.8 \mathrm{t} \mathrm{CO}_{2}$ $\mathrm{e} \mathrm{ha}^{-1}$ year $^{-1}$, respectively, in Nacharam, Yagantipalli and Chamaluru villages. Among the eight villages studied, Kurnool and Nacharam had a negative $\mathrm{C}$ balance of about -3002 and $-2450 \mathrm{t} \mathrm{CO}_{2}$ e (high sink) when compared to other study villages. Although Yagantipalle had a less area under perennial crops (55 ha), it had maximum mitigation potential compared to Nacharam (217.4 ha) due to cultivation of perennial fodder grasses compared to subabul cultivation in Nacharam.

\section{Non-forest land use change}

The conversion of land use from one to another production systems results in the emissions or mitigation of $\mathrm{CO}_{2}$ from biomass and soils, as well as $\mathrm{N}_{2} \mathrm{O}$ and $\mathrm{CH}_{4}$ emissions from the soil. The non-forest land use change module in EXACT model projected $\mathrm{CO}_{2}$ emissions (Table 6). Nacharam where 80.5 ha of waste lands was converted into annual crops under Indira Jala Prabha scheme has shown the $\mathrm{CO}_{2}$ emissions of $3566 \mathrm{t} \mathrm{CO}_{2}$ e. In Sirusuwada, 35 ha of waste lands are converted into sugarcane crop, which resulted in $\mathrm{CO}_{2}$ emissions of $3042 \mathrm{t} \mathrm{CO}_{2}$ e. Paddy area (irrigated) was converted into sorghum crop due to water scarcity in Chakrayapet, and this resulted in $\mathrm{CO}_{2}$ emissions of $1600 \mathrm{t}$ $\mathrm{CO}_{2}$ e according to the model predictions. Among the study villages, Srikakulam had maximum positive $\mathrm{C}$ balance (source), followed by Nacharam, while Yagantipalle had negative carbon balance as depicted in Fig. 3 . 
Table 7 Emissions ( $\mathrm{C}_{2}$ e) from livestock by implementation of climate smart management practices in livestock

\begin{tabular}{|c|c|c|c|c|c|}
\hline Village & $\begin{array}{l}\mathrm{CH}_{4} \text { from enteric } \\
\text { fermentation }\end{array}$ & $\begin{array}{l}\mathrm{CH}_{4} \text { from manure } \\
\text { management }\end{array}$ & $\begin{array}{l}\mathrm{N}_{2} \mathrm{O} \text { from manure } \\
\text { management }\end{array}$ & $\begin{array}{l}\text { Improved feeding } \\
\text { practices }\end{array}$ & $\begin{array}{l}\text { Final } \\
\text { balance }\end{array}$ \\
\hline Chamaluru & 9044 & 407 & 4131 & -608 & 12,975 \\
\hline Chakrayapet & 343 & 27 & 100 & -75 & 395 \\
\hline Nacharam & 1222 & 111 & 184 & -55 & 1461 \\
\hline Yagantipalli & 3694 & 343 & 469 & -462 & 4045 \\
\hline Sirusuwada & 1979 & 171 & 457 & -292 & 2315 \\
\hline Matsyapuri & 0 & 0 & 0 & -340 & -340 \\
\hline Nandyalagudem & 318 & 23 & 116 & -74 & 383 \\
\hline Boringthanda & 814 & 75 & 199 & -134 & 954 \\
\hline
\end{tabular}

- Carbon sink and + carbon source

Table $8 \mathrm{CO}_{2}$ emissions $\left(\mathrm{t} \mathrm{CO}_{2}\right.$ e) due to use of fertilizers and agricultural chemicals in study villages

\begin{tabular}{|c|c|c|c|c|c|c|c|c|c|}
\hline \multirow[t]{2}{*}{ Village } & \multicolumn{2}{|c|}{$\begin{array}{l}\mathrm{CO}_{2} \text { emission from } \\
\text { urea }\end{array}$} & \multicolumn{2}{|c|}{$\begin{array}{l}\mathrm{N}_{2} \mathrm{O} \text { emissions from } \\
\text { urea }\end{array}$} & \multicolumn{2}{|c|}{$\begin{array}{l}\mathrm{CO}_{2} \text { eq. emission from production transport } \\
\text { transfer of chemicals and } \mathrm{P} \text { and } \mathrm{K} \text { fertilizers }\end{array}$} & \multicolumn{2}{|c|}{ Total emission } & \multirow[t]{2}{*}{ Balance } \\
\hline & Without & With & Without & With & Without & With & Without & With & \\
\hline Nacharam & 1344 & 1564 & 25,225 & 26,820 & 29,400 & 30,149 & 55,969 & 58,533 & 2564 \\
\hline Nandyalagudem & 294 & 249 & 9000 & 7658 & 9263 & 8161 & 18,557 & 16,068 & -2489 \\
\hline Boringthanda & 163 & 137 & 5180 & 4393 & 5342 & 4615 & 10,685 & 9144 & -1541 \\
\hline Yagantipalli & 388 & 284 & 17,540 & 13,144 & 22,044 & 16,676 & 39,972 & 30,104 & -9868 \\
\hline Matsyapuri & 616 & 584 & 11,617 & 10,631 & 24,877 & 20,634 & 37,110 & 31,849 & -5261 \\
\hline Sirusuwada & 117 & 89 & 3595 & 3015 & 4132 & 3822 & 7844 & 6926 & -918 \\
\hline Chamaluru & 1423 & 895 & 16,174 & 10,170 & 22,589 & 14,709 & 40,456 & 25,774 & $-14,682$ \\
\hline Chakrayapet & 114 & 72 & 1296 & 815 & 1831 & 1179 & 3242 & 2065 & -1176 \\
\hline Min & 114 & 72 & 1296 & 815 & 1831 & 1179 & 3242 & 2065 & $-14,682$ \\
\hline Max & 1423 & 1564 & 25,225 & 26,820 & 29,400 & 30,149 & 55,969 & 58,533 & 2564 \\
\hline Mean & 557 & 484 & 11,203 & 9581 & 14,935 & 12,493 & 26,729 & 22,558 & -4171 \\
\hline SD & 537 & 519 & 8096 & 8123 & 10,888 & 9888 & 19,120 & 18,261 & 5591 \\
\hline $\mathrm{CV}$ & 96 & 107 & 72 & 85 & 73 & 79 & 72 & 81 & -134 \\
\hline
\end{tabular}

- Carbon sink and + carbon source

\section{Livestock and small ruminant interventions}

The livestock and small ruminants contribute both directly and indirectly to climate change through the emissions of greenhouse gases. Globally, the sector contributes 18 percentage (7.1 billion tons $\mathrm{CO}_{2}$ equivalent) of greenhouse gas emissions (http://www.fao.org/agriculture). In our study, it was observed that livestock sector is a major source of $\mathrm{CO}_{2}$ emissions through enteric fermentation, apart from $\mathrm{N}_{2} \mathrm{O}$ emissions from manure management (Table 7). In Chamaluru village, the number of livestock and small ruminants were greater as crops often suffer due to prevailing drought conditions in this village; livestock is major production system; and more than 10,000 sheep, followed by goats and buffaloes, were maintained in the village "with" project, which resulted in $\mathrm{CO}_{2}$ emissions by 13,403 $\mathrm{t} \mathrm{CO}_{2}$ e (Table 8). However, feeding of improved fodder such as Co-1, Co-5, napier grass and breeding practices mitigate the $\mathrm{CO}_{2}$ emissions and resulted in reduction in $\mathrm{CO}_{2}$ emissions by only $4.5 \%$. In Yagantipalli, buffalos were the main livestock that resulted in methane emissions of $3694 \mathrm{t} \mathrm{CO}_{2}$ e through enteric fermentation "with" project and finally created a $\mathrm{C}$ balance of $4045 \mathrm{t} \mathrm{CO}_{2} \mathrm{e}$. However, $9.3 \%$ of $\mathrm{CO}_{2}$ emissions were mitigated "with" project through improved feeding and breeding practices. As there was no more increase in number of livestock 
Table 9 Relationship of carbon balance with different parameters in different study villages

\begin{tabular}{lll}
\hline Relationship of parameters in the model & Regression model & $R^{2}$ \\
\hline Livestock and small ruminants versus C balance & $\mathrm{C}$ bal $=1.163$ (LS) +279.8 & $0.858^{*}$ \\
Total area of annual crops versus total C balance & $\mathrm{C}$ bal $=-25.77(\mathrm{~A})-391.7$ & $\mathrm{C}$ bal $=-25.76(\mathrm{MA})-95.39$ \\
Mean area of annual crops versus total C balance & $\mathrm{CH}_{4}$ emissions $=-10.69(\mathrm{C}$ bal) $-10,744$ & $0.812^{*}$ \\
Methane emitted in rice-growing season versus carbon balance (with project) & $\mathrm{CH}_{4}$ emissions $=-11.69(\mathrm{C}$ bal) $-10,744$ & $0.838^{*}$ \\
Methane emitted in rice-growing season versus carbon balance (without project)
\end{tabular}

$L S$ livestock and small ruminants, $C$ bal Carbon balance, $A$ area, $M A$ mean area, GHG mit GHG mitigation, Mit pot mitigation potential

* and ** indicate significance at $p<0.05$ and $p<0.01$ level, respectively

"with" project in Matsyapuri village, the improved breeding and feeding methods practiced resulted in mitigation of $\mathrm{CO}_{2}$ emissions by $-340 \mathrm{t} \mathrm{CO}_{2}$ e.

\section{Fertilizer management}

All the chemical fertilizers do not have the same $\mathrm{CO}_{2}$ emission potential, which depends on the molecule that contains $\mathrm{N}$ and $\mathrm{P}$ in different proportion. Hence, the EX-ACT module "inputs" takes into account the expected $\mathrm{CO}_{2}$ emissions due to production, transportation and application of fertilizers as shown by the corresponding default coefficients proposed by IPCC and used in the EX-ACT estimations (Table 8).

The fertilizers use in the study villages before the project initiation did not follow the soil test values and resulted in higher $\mathrm{CO}_{2}$ emissions. The nutrient management practices such as soil-test-based fertilizer application and integrated nutrient management resulted in the mitigation of $\mathrm{CO}_{2}$ emissions. The results showed that in Chamaluru, "without" project, the $\mathrm{CO}_{2}$ emissions were $4046 \mathrm{t} \mathrm{CO}_{2} \mathrm{e}$ and, "with" project, the emissions were $25,774 \mathrm{t} \mathrm{CO}_{2} \mathrm{e}$, of which $3.45 \%$ of $\mathrm{CO}_{2}$ emissions and $39.5 \%$ of $\mathrm{N}_{2} \mathrm{O}$ emissions were due to application of urea and $57.0 \%$ emissions were due to its production and transportation. Similarly, in Yagantipalli, with project interventions, $0.9 \%$ of $\mathrm{CO}_{2}$ emissions and $46.7 \%$ of $\mathrm{N}_{2} \mathrm{O}$ emissions were due to application of urea and $55.4 \%$ emissions were due to its production and transportation. Adopting the improved nutrient management practices management resulted in mitigation of $\mathrm{CO}_{2}$ emissions by $-14,682,-9848$, -5261 and -2489 t $\mathrm{CO}_{2} \mathrm{e}$ in Chamaluru, Yagantipalli, Matsyapuri and Nandyalagudem, respectively.

In Nacharam, without the project, the use of fertilizers was very low and the use of potassium fertilizers was totally avoided. With the interventions of the project, due to soil-test-based fertilizer recommendations, the use of nitrogen and potassium fertilizers increased and this resulted in total $\mathrm{CO}_{2}$ emissions of 58,533 $\mathrm{t} \mathrm{CO}_{2} \mathrm{e}$, which is high when compared without the project $\left(55,969 \mathrm{t} \mathrm{CO}_{2} \mathrm{e}\right)$; and this in turn created $\mathrm{C}$ balance of $2564 \mathrm{t} \mathrm{CO}_{2} \mathrm{e}$ (Table 8). In most of the situations, the $\mathrm{CO}_{2}$ emission was ranging between 0 and 22,000 $\mathrm{t} \mathrm{CO}_{2} \mathrm{e}$.

\section{Relationship of $\mathbf{C}$ balance with livestock and small ruminants, area under annual crops and methane emissions in irrigated rice with $\mathrm{C}$ balance}

The study indicated that the total $\mathrm{C}$ balance was significantly influenced with an increase in the total livestock and small ruminants at different locations. Most of the locations had a livestock + ruminant population up to 2000 units, while it was a maximum of about 10,540 units in Chamaluru in Anantapur district due to a large sheep population maintained by the farmers. The positive $\mathrm{C}$ balance in terms of $\mathrm{CO}_{2}$ emissions was found to be mainly due to enteric fermentation which resulted in the methane emission that is 21 times equivalent to $\mathrm{CO}_{2}$ emission. The partial regression coefficient of the model fitted for the carbon data as influenced by the emissions due to number of livestock and small ruminants indicated that the rate of change in $\mathrm{C}$ balance in terms of $\mathrm{CO}_{2}$ emissions is of the magnitude of 1.163 units for a unit change in livestock and ruminant population. The regression model gave good predictability of carbon $\left(R^{2}=0.858\right)$ as influenced by the livestock + ruminant population.

Regression model revealed that with an increase in the total area of different annual crops, the total carbon balance in terms of $\mathrm{CO}_{2}$ emissions was found to be less (negative) under the traditional system, where its degree was further reduced under the improved system (with adoption climate smart practices). The $R^{2}$ value was found to be significant and higher $\left(R^{2}=0.997\right)$ under improved practice, while it 
was 0.896 under the traditional practice for different crops at different study villages.

In irrigated rice, the relationship between $\mathrm{C}$ balance and area (ha) was found to be significant with $R^{2} \geq 0.768$ (Table 9). By and large, area under irrigated rice was between 0 and 150 ha in different locations except in Matsyapuri, where the area was maximum since rice is the only crop at this study village. The important management practice to reduce the methane emissions from rice crop is intermittent flooding instead of continuous flooding. Although all the villages followed intermittent flooding during rice cultivation, two villages showed $\mathrm{CO}_{2}$ emissions mainly due to the residue burning, while the rest followed residue incorporation or FYM application and green leaf manuring along with nutrient management. From the regression model, it was found that with intermittent flooding, nutrient management and addition of organics, unit area increased under irrigated rice could mitigate the $\mathrm{CO}_{2}$ emissions by about -11.93 units.

\section{Final balance in six districts}

The results of EX-ACT model in eight villages showed mitigation of $\mathrm{CO}_{2}$ emissions with different climate smart management practices (Table 3). Earlier, it is reported that the improved agronomic practices increased yields by generating higher inputs of $\mathrm{C}$ through residues that in turn improve soil organic C. Such practices include improved crop varieties, crop rotation, water management, nutrient and manure management (Alvarez 2005), and livestock management increase soil $\mathrm{C}$ gains similarly, growing perennial crops and afforestation can mitigate the $\mathrm{CO}_{2}$ emissions.

\section{Improved annual crop management practices}

The results of our study for annual crops showed that with climate smart (improved) management practices, $\mathrm{CO}_{2}$ emissions were mitigated by reducing its addition to the atmosphere. In Nandyalagudem and Nacharam villages, 63.4 and $80 \%$ of $\mathrm{CO}_{2}$ mitigation resulted from annual crops with interventions such as residue recycling instead of residue burning. Also, it is reported that improved agronomic practices (improved varieties, balanced use of fertilizer based on soil testing, green manure incorporation and reduced tillage practices) result in generation of positive effects in terms of increased carbon stock in soil (Branca et al. 2013; Srinivasarao et al. 2013). Incorporation of crop residue into the soil with rotavator helped in mitigating the $\mathrm{CO}_{2}$ emissions by building up SOC which in turn improves the availability of nutrients such as nitrogen, phosphorus, sulfur and potassium and making them available to plant roots. Even reduced tillage would provide minimum soil disturbance and improve the soil aggregation through enhanced C sequestration (Jabro et al. 2008). The rate of SOC sequestration was greater with regular application of organic matter; application of chemical fertilizers and conjunctive use of organic and mineral fertilizers significantly enhance SOC stock (Srinivasarao et al. 2012).

\section{Improved or water saving paddy systems}

From our study, it was observed that mitigation of $\mathrm{CH}_{4}$ and $\mathrm{N}_{2} \mathrm{O}$ emissions in terms of $\mathrm{CO}_{2}$ emissions from the rice fields was possible by following intermittent flooding instead of continuous flooding along with green manure incorporation or straw incorporation into the field (Table 4). The extent of methane mitigation varies depending on soil type, extent of drainage and frequency of drainage. It is reported that the decomposition of straw in rice field resulted in reducing the $\mathrm{N}_{2} \mathrm{O}$ emissions by suppressing nitrogen mineralization (Wang et al. 2011; Ma et al. 2010) and thereby suppressing nitrification- and denitrificationassociated $\mathrm{N}_{2} \mathrm{O}$ emission. Similar results were observed in rice-growing villages where $\mathrm{N}_{2} \mathrm{O}$ emissions decreased due to incorporation of straw and green manure into the soil. It is well documented that the effect of straw application on $\mathrm{N}_{2} \mathrm{O}$ emission in rice fields was negatively related to the straw application rate, and oxygen depletion might be responsible for low $\mathrm{N}_{2} \mathrm{O}$ emissions in irrigated paddy fields (Kreye et al. 2007).

\section{Afforestation and perennial crops}

Afforestation and perennial plantation caused substantial decrease in $\mathrm{CO}_{2}$ emissions. By adopting the plantation of perennial crops in an area about 24,29 and 16 ha in Nacharam, Yagantipalli and Chamaluru resulted in the mitigation potential of $12.4,12.9$ and $8.1 \%$, respectively. Perennial crops improve the soil condition (Mutuo et al. 2005) and would enhance the C sequestration (Dixon 1995) by improving crop productivity, thereby reducing soil loss by erosion. The land use change through afforestation or reforestation should increase $\mathrm{C}$ sequestration per unit of land, and the rate of $\mathrm{C}$ sequestered by trees within a system will depend on tree species, age and density (Quinkenstein et al. 2009) besides the 
edapho-climatic conditions, management, fertilization and land clearing. Litter falling from plants and dead roots from plant material are decomposed into soil C. Some of the soil C would be taken up by microbes and stored, and the remaining $\mathrm{C}$ would become mineralized. Soil would store $\mathrm{C}$, but as a result of the mineralization and root respiration, part of that $\mathrm{C}$ is released back to the atmosphere. Afforestation showed mitigation potential of $\mathrm{CO}_{2}$ emissions at the rate of 8.2 and $16.8 \%$ in Chamaluru and Chakrayapet. Branca et al. (2013) highlighted that the conversion of degraded land into perennial crops will cause an increase in soil organic $\mathrm{C}$ stock in the Rio Rural project area from 15.5 to $47.0 \mathrm{tC} \mathrm{ha}^{-1}$.

\section{Non-forest land use change}

Conversion of wastelands or fallow lands into cultivated lands resulted in $\mathrm{CO}_{2}$ emission. In fallow lands, accumulation of organic matter is high and conversion of these lands resulted in decrease in the carbon stock. This is due to soil degradation, erosion and leaching of soil nutrients. The conversion of annual crops to perennial crops such as horticultural crops and annual crops converted into fodder crops resulted in mitigation of $\mathrm{CO}_{2}$ emission. The perennial crops would increase the underground $\mathrm{C}$ storage (Bernoux et al. 2001). It is also important to explore and consider technologies and practices that could lead to minimizing the $\mathrm{CO}_{2}$ emissions during land conversion.

\section{Livestock interventions}

Improved feeding practices would mitigate the methane emissions, and the mitigation options associated with reduced enteric methane are associated with improved feed use by the animals. Adopting improved feeding practices such as green fodder, mineral mixtures and mixtures of byproducts of crops (pigeon pea, maize) and breeding practices has mitigated the methane emissions by $-608,-55$, -462 and -292 t CO$_{2}$ e (Table 7). Methane emissions were determined by animal type, quantity and quality of feed, animal body weight, age and amount of exercise by the animal. Smith et al. (2007) showed that the use of higher level of concentrates may increase $\mathrm{CH}_{4}$ emissions per animal, but also increase productivity (meat and milk), thus resulting in an overall reduction in $\mathrm{CH}_{4}$ emissions per unit of product. Quality feed reduce $\mathrm{CH}_{4}$ production by altering the composition of volatile fatty acids produced. Methane emissions are reduced potentially with fodder high in soluble carbohydrates (Eckard et al. 2000; Monteny et al. 2006).

\section{Fertilizer management}

Nitrogen fertilizers applied to crops is not used efficiently. The practices followed to improve the $\mathrm{N}$ use efficiency and to reduce $\mathrm{N}_{2} \mathrm{O}$ emissions include the use of slow release of nitrogen fertilizers, method and time of application and avoiding the use of excess $\mathrm{N}$ fertilizers (Monteny et al. 2006).

The practices followed by farmers in study villages such as use of fertilizers based on soil testing, use of organic manures and application of fertilizer $\mathrm{N}$ based on leaf color charts resulted in decrease in emissions of $\mathrm{N}_{2} \mathrm{O}$, which is 298-fold damageable than $\mathrm{CO}_{2}$ emissions (Table 8). Nitrous oxide is an intermediate product of the soil processes of nitrification and denitrification and is affected by moisture, temperature, mineral $\mathrm{N}$ content, available soil carbon and $\mathrm{pH}$. Apart from management practices such as agronomic and water management, $\mathrm{N}$ fertilizer application influenced $\mathrm{N}_{2} \mathrm{O}$ production (Dobbie and Smith 2003). Studies have shown that the increased use of $\mathrm{N}$ fertilizers potentially result in increased $\mathrm{N}_{2} \mathrm{O}$ emissions from soils as a result of increased mineral $\mathrm{N}$ content (Smith et al. 1997).

Adopting proper nutrient management practices such as reducing the amount of $\mathrm{N}$ fertilizers use, balanced fertilizer use, split application of $\mathrm{N}$, placement of fertilizers with seed-cum-fertilizer and drilling of $\mathrm{N}$ fertilizer introduced in the villages resulted in mitigation of $\mathrm{CO}_{2}$ emissions. In addition, the use of crop residues and legume green manures was practiced to meet partial $\mathrm{N}$ requirements in annual and vegetable crops. Improved $\mathrm{N}$ use efficiency resulted from balance nutrition and water management in case of irrigated rice has contributed to negative carbon balance.

\section{Conclusion}

To assess the carbon balance in selected districts, different climate smart management practices including annual, perennial, no forest land use change, irrigated rice, inputs and livestock were taken into account. The model projections regarding the impact of project activities on $\mathrm{CO}_{2}$ emissions demonstrated that the mitigation benefits was achieved through adoption of climate smart agricultural 
practices at the village level. With the implementation of climate smart agricultural practices proposed by the NICRA project, the EX-ACT model projected overall negative $C$ balance in different study villages. Even in village like Matsyapuri, where paddy is the predominant cropping system organic amendments and water management could reduce $\mathrm{CO}_{2}$ emission impact. EX-ACT as a tool was successful in quantifying the mitigation potentials of $\mathrm{CO}_{2}$ emissions in diverse situation in the villages of different districts of Andhra Pradesh, India.

Acknowledgements We sincerely thank the Indian Council of Agricultural Research (ICAR) for funding under National Initiative on Climate Resilient Agriculture (ICAR-NICRA: 4-3/2010-1.A.II) and Central Research Institute for Dryland Agriculture (CRIDA) for providing all the facilities. Authors would like to thank anonymous reviewers whose suggestions have contributed to the improvement of the manuscript significantly.

\section{References}

Alvarez R (2005) A review of nitrogen fertilizer and conservative tillage effects on soil organic storage. Soil Use Manag 21:38-52

Bernoux M, Da Conceicao Maria, Carvalho Santana, Volkoff Boris, Cerri Arlos Clemente (2001) $\mathrm{CO}_{2}$ emission from mineral soils following land-cover change in Brazil. Glob Change Biol 7:779-787

Bernoux M, Bockel L, Branca G, Tinlot M (2010) EX-ante carbonbalance tool (EXACT) technical guidelines, version May 2010, EASYPol Module 101, FAO, Rome, p 79. http://www.fao.org/ docs/up/easypol/780/ex-act-tech-guidelines_101en.pdf

Bhatia A, Pathak H, Jain N, Singh PK, Singh AK (2005) Global warming potential of manure amended soils under rice-wheat system in the Indo-Gangetic plains. Atmos Environ 39:6976-6984

Branca G, Hissa H, Benez MC, Medeiros K, Lipper L, Tinlot M, Bockel L, Bernoux M (2013) Capturing synergies between rural development and agricultural mitigation in Brazil. Land Use Policy 30:507-518

Dixon RK (1995) Agroforestry systems: sources or sinks of greenhouse gases? Agrofor Syst 31:99-116

Dobbie KE, Smith KA (2003) Impact of different forms of $\mathrm{N}$ fertilizer on $\mathrm{N}_{2} \mathrm{O}$ emissions from intensive grassland. Nutr Cycl Agroecosyst 67:37-46

Eckard RJ, Dalley D, Crawford M (2000) Impacts of potential management changes on greenhouse gas emissions and sequestration from dairy production systems in Australia. In: Bugg AL, Ainslie H, Keenan R (ed) Management options for Carbon Sequestration in forest, agricultural and rangeland ecosystems, Workshop Proceedings. ANU, Canberra. pp 58-72. CRC for Greenhouse Accounting. ISBN 0646404318

Jabro JD, Sainju U, Stevens WB, Evans RG (2008) Carbon dioxide flux as affected by tillage and irrigation in soil converted from perennial forages to annual crops. J Environ Manag 88:1478-1484

Jordan E, Lovett DK, Hawkins M, Callan J, O'Mara FP (2006) The effect of varying levels of coconut oil on intake, digestibility and methane output from continental cross beef heifers. J Anim Sci 82:859-865

Kreye C, Dittert K, Zheng XH, Zhang X, Lin S, Tao HB, Sattelmacher B (2007) Fluxes of methane and nitrous oxide in water-saving rice production in north China. Nutr Cycl Agroecosyst 77:293-304

Lal R (2004) Soil carbon sequestration in India. Clim Change 65:277-296

Lal R (2009) Soil carbon sequestration for climate change mitigation and food security. In Souvenir, Platinum Jubilee Symposium on Soil science in meet. The challenges to food security and environmental quality, New Delhi, 22-25 December. Indian Society of Soil Science, New Delhi, pp 39-46

Lal R (2010) Carbon sequestration potential of rainfed agriculture. Indian. J Dryland Agric Res Dev 25:1-6

Ma E, Zhang G, Ma J, Xu H, Cai Z, Yagi K (2010) Effects of rice straw returning methods on $\mathrm{N} 2 \mathrm{O}$ emissions during wheatgrowing season. Nutr Cycl Agroecosyst 88:463-469

Mandal B, Majumdar B, Bandhopadhyay PK, Hazra GC, Gangopadhyay A, Samantaray RN, Mishra AK, Chaudhury J, Saha MN, Kundu S (2007) The potential cropping systems and soil amendments for carbon sequestration in soils under long-term experiments in subtropical India. Glob Change Biol 13:357-369

Monteny GJ, Bannink A, Chadwick D (2006) Greenhouse gas abatement strategies for animal husbandry. Agric Ecosyst Environ 112:163-170

Mutuo PK, Cadisch G, Albrecht A, Palm CA, Verchot L (2005) Potential of agroforestry for carbon sequestration and mitigation of greenhouse gas emissions from soils in the tropics. Nutr Cycl Agroecosyst 71:43-54

Quinkenstein A, Wollecke J, Bohm C, Grunewald H, Freese D, Schneider BU, Huttle RF (2009) Ecological benefits of the alley cropping agroforestry system in sensitive regions of Europe. Environ Sci Policy 12(8):1112-1121

Rajamani L (2007) India's negotiating position on climate change: legitimate but not sagacious. CPR issue brief. Number II. www. cprindia.org

Smith KA, McTaggart IP, Tsuruta H, Smith K (1997) Emissions of $\mathrm{N}_{2} \mathrm{O}$ and $\mathrm{NO}$ associated with nitrogen fertilization in intensive agriculture, and potential for mitigation. Soil Use Manag 13:296-304

Smith P, Martino D, Cai Z, Gwary D, Janzen HH, Kumar P, Mccarl B, Ogle S, O'mara F, Rice C, Scholes RJ, Sirotenko O (2007) Agriculture. Chapter 8. In: Metz B, Davidson OR, Bosch PR, Dave R, Meyer A (eds) Climate Change 2007: Mitigation. Contribution of Working Group III to the Fourth Assessment Report of the Intergovernmental Panel on Climate Change, Cambridge University Press, Cambridge, United Kingdom and New York, NY, USA

Srinivasarao Ch, Venkateswarlu B, Dixit S, Kundu S, Gayatri Devi K (2011a) Livelihood impacts of soil health improvement in backwards and tribal districts of Andhra Pradesh. Central Research Institute for Dryland Agriculture, Hyderabad

Srinivasarao Ch, Venkateswarlu B, Srinivas K, Kundu S, Singh AK (2011b) Soil carbon sequestration for climate change mitigation and food security, 24th November-3rd December, 2011. Central Research Institute for Dryland Agriculture, Hyderabad, p 322

Srinivasarao Ch, Venkateswarlu B, Dinesh Babu M, Wani SP, Dixit Sreenath, Sahrawat KL, Kundu Sumanta (2011c) Soil health improvement with glyricidia green leaf manuring in rainfed agriculture. Central Research Institute for Dryland Agriculture, Hyderabad, p 23

Srinivasarao Ch, Venkateswarlu B, Lal R, Singh AK, Vittal KPR, Kundu Sumanta, Singh SR, Singh SP (2012) Long-term effects of soil fertility management on carbon sequestration in a ricelentil cropping system of the Indo-Gangetic plains. Soil Sci Soc Am J 76:168-178

Srinivasarao Ch, Venkateswarlu B, Lal Rattan, Singh AK, Kundu Sumantha (2013) Sustainable management of soils of dryland 
ecosystems of India for enhancing agronomic productivity and sequestering carbon. Adv Agron 121:253-329

Sundermeier A, Reeder R, Lal R (2005) Soil Carbon SequestrationFundamentals. Extension Factsheet AEX-510-05, Ohio State University Extension pp 510-505

Wang JY, Jia JX, Xiong JQ, Khalil MAK, Xing GX (2011) Water regime-nitrogen fertilizer-straw incorporation interaction: field study on nitrous oxide emissions from a rice agroecosystem in Nanjing, China. Agric Ecosyst Environ 141:437-446

Wright AL, Hons FM (2005) Tillage impacts on soil aggregation and carbon and nitrogen sequestration under wheat cropping sequences. Soil Tillage Res 84:67-75 